\title{
Comparative transcriptome profiling and co-expression network analysis uncover the key genes associated withearly-stage resistance to Aspergillus flavus in maize
}

Huanhuan Liư ${ }^{\dagger}$, Haofeng Wu${ }^{\dagger}$, Yan Wang, Huan Wang, Saihua Chen ${ }^{*}$ and Zhitong Yin ${ }^{*}$

\begin{abstract}
Background: The fungus Aspergillus flavus (A. flavus) is a serious threat to maize (Zea mays) production worldwide. It causes considerable yield and economic losses, and poses a health risk to humans and livestock due to the high toxicity of aflatoxin. However, key genes and regulatory networks conferring maize resistance to $A$. flavus are not clear, especially at the early stage of infection. Here, we performed a comprehensive transcriptome analysis of two maize inbred lines with contrasting resistance to A. flavus infection.

Results: The pairwise comparisons between mock and infected kernels in each line during the first $6 \mathrm{~h}$ post inoculation (hpi) showed that maize resistance to A. flavus infection was specific to the genotype and infection stage, and defense pathways were strengthened in the resistant line. Further comparison of the two maize lines revealed that the infection-induced up-regulated differentially expressed genes (DEGs) in the resistant line might underlie the enhanced resistance. Gene co-expression network analysis by WGCNA (weighted gene co-expression network analysis) identified 7 modules that were significantly associated with different infection stages, and 110 hub genes of these modules. These key regulators mainly participate in the biosynthesis of fatty acid and antibiotics. In addition, 90 candidate genes for maize resistance to A. flavus infection and/or aflatoxin contamination obtained in previous studies were confirmed to be differentially expressed between the resistant and susceptible lines within the first 6 hpi.
\end{abstract}

Conclusion: This work unveiled more A. flavus resistance genes and provided a detailed regulatory network of early-stage resistance to $A$. flavus in maize.

Keywords: Maize, Aspergillus flavus, Transcriptome analysis, WGCNA, Early-stage resistance

\footnotetext{
*Correspondence: chensaihua@yzu.edu.cn; ztyin@yzu.edu.cn

${ }^{\dagger}$ Huanhuan Liu and Haofeng Wu contributed equally to this work.

Jiangsu Key Laboratory of Crop Genetics and Physiology/Co-Innovation Center for Modern Production Technology of Grain Crops/Key Laboratory of Plant Functional Genomics of the Ministry of Education/Joint International Research Laboratory of Agriculture \& Agri-Product Safety of the Ministry of Education, Yangzhou University, Yangzhou 225009, China
}

(c) The Author(s). 2021 Open Access This article is licensed under a Creative Commons Attribution 4.0 International License, which permits use, sharing, adaptation, distribution and reproduction in any medium or format, as long as you give appropriate credit to the original author(s) and the source, provide a link to the Creative Commons licence, and indicate if changes were made. The images or other third party material in this article are included in the article's Creative Commons licence, unless indicated otherwise in a credit line to the material. If material is not included in the article's Creative Commons licence and your intended use is not permitted by statutory regulation or exceeds the permitted use, you will need to obtain permission directly from the copyright holder. To view a copy of this licence, visit http://creativecommons.org/licenses/by/4.0/ The Creative Commons Public Domain Dedication waiver (http://creativecommons.org/publicdomain/zero/1.0/) applies to the data made available in this article, unless otherwise stated in a credit line to the data. 


\section{Background}

Maize is a major staple food and feed crop in the world and is vulnerable to various phytopathogens. The fungus A. flavus can infect maize before and after harvest, leading to ear and kernel rot and subsequent contamination with aflatoxins [1]. Aflatoxins are highly toxic and carcinogenic, and mainly damage the liver tissues of humans and animals. It is estimated that $4.6-28.2 \%$ of liver cancers worldwide are caused by long-term excessive intake of aflatoxins [2]. Breeding resistant maize cultivars is regarded as the most cost-effective measure for controlling the damage of $A$. flavus. Since resistance to $A$. flavus is a complex quantitative trait [3, 4], molecular techniques, including marker-assisted selection (MAS), transgenic breeding, and gene editing, will facilitate the development of resistant cultivars. However, exploring resistance genes and gene regulatory networks is a prerequisite for the molecular breeding for maize resistance to A. flavus.

In the past two decades, quantitative trait locus (QTL) analysis has been widely used to map the genes conveying maize resistance to $A$. flavus infection and aflatoxin accumulation. Numerous QTLs that explained no more than $20 \%$ of the phenotypic variation using linkage mapping methods have been reported [5-13]. In addition, many single nucleotide polymorphisms associated with maize resistance have been identified through genome-wide association studies (GWAS) [14-16]. Up to now, no genes associated with $A$. flavus infection and aflatoxin accumulation in maize have been cloned through forward genetics approaches. Whereas, several genes or proteins related to maize defense have been identified and characterized by quantitative PCR (qPCR), RNA interference (RNAi), and other methods. For example, pathogenesis-related (PR) proteins, such as PR10 [17], chitinase [18], PRm [19], and lipoxygenase [20], were reported to be involved in host pathogen recognition and susceptibility to $A$. flavus infection and aflatoxin accumulation. Some proteins in the maize signaling pathway, such as calcium-dependent protein kinases, respiratory burst oxidases, and WRKY family transcription factors, function primarily in regulating the expression of antioxidant and $P R$ gene expression [21]. Other factors, including phytohormones and polyamines, are also considered to be important factors in the regulation of maize resistance to A. flavus. For example, the increase of ethylene content in maize kernels contributed to the proliferation of A. flavus [22]. Further, polyamine metabolism facilitates aflatoxin resistance and overall stress tolerance in maize [23]. Though specific genes and pathways have been linked to maize resistance, a detailed description of the transcriptome dynamics and transcriptional networks during fungal infection deserves further investigation.
Transcriptome analyses based on microarray and RNA sequencing (RNA-seq) technologies can provide crucial systems-level insight into the transcriptional network of pathogen infection. Many differentially expressed genes (DEGs) and signaling pathways have been confirmed to be involved in the regulation of resistance to A. flavus by several maize transcriptome studies [24-27]. Recently, dual RNA-seq analyses have been used to generate gene co-expression networks, including both maize and $A$. flavus genes, to better understand the complex interaction between the two organisms [28, 29]. However, most of these studies focused on the dynamic changes of gene expression over several days after $A$. flavus inoculation, but not to the early stage of plant-pathogen interactions. Previous studies have demonstrated that the pathogen infection always triggers a rapid response in the initial stage. At as early as $3 \mathrm{~h}$ post inoculation (hpi), $Z m C C T$, the causal gene of a quantitative diseaseresistance locus against stalk rot in maize, reached its highest expression level [30]. In the first $6 \mathrm{hpi}$, candidate genes underlying resistance to Fusarium ear rot have been identified to be differentially expressed between resistant and susceptible maize lines [31]. At 1 hpi with Phytophthora infestans, seven potato (Solanum tuberosum) pathogenesis/defense-related genes were more highly induced in the resistant cultivar than in the susceptible cultivar [32]. The dynamic changes in the early stage of $A$. flavus infection are still elusive. Therefore, it is necessary to analyze the transcriptome of maize kernels in the early stage of $A$. flavus infection.

In this study, we compared and analyzed the dynamic transcriptome reprogramming of two maize inbred lines with contrasting resistance to $A$. flavus. To identify early-stage response genes and gene co-expression networks associated with maize resistance to $A$. flavus, the kernels were sampled at $0,0.5,1.5,3$, and 6 hpi. Key regulatory mechanisms that determine maize resistance were identified using an integrated analysis of DEGs and co-expression networks. The expression patterns of previously discovered candidate genes that confer maize resistance were also investigated. The results of our study provide insights into the key factors and molecular mechanisms underlying the resistance of maize to $A$. flavus infection.

\section{Results}

Evaluation of maize inbred lines after inoculation with $A$. flavus

Two maize inbred lines, AF99 and AF32, were selected to investigate resistance to $A$. flavus by inoculating kernels with conidia at pre- and post-harvest time points (Fig. 1). In the pre-harvest inoculation, the A. flavus infection was mainly restricted to the inoculated dot in AF99, while it expanded into many other kernels and 
a

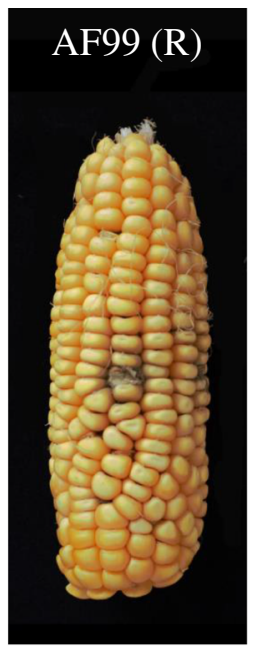

$\mathrm{b}$

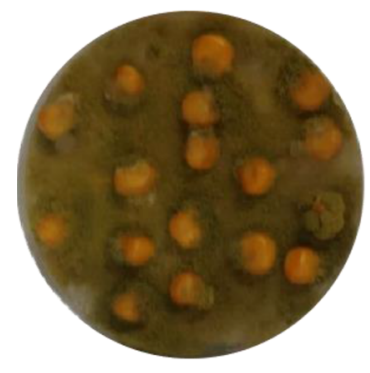

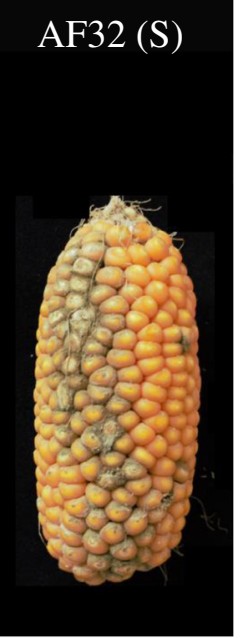

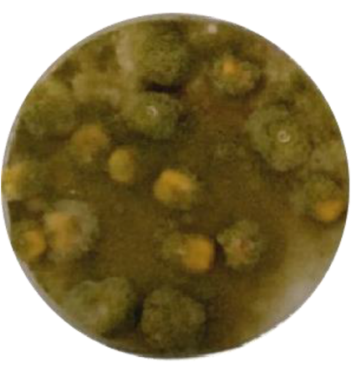

C
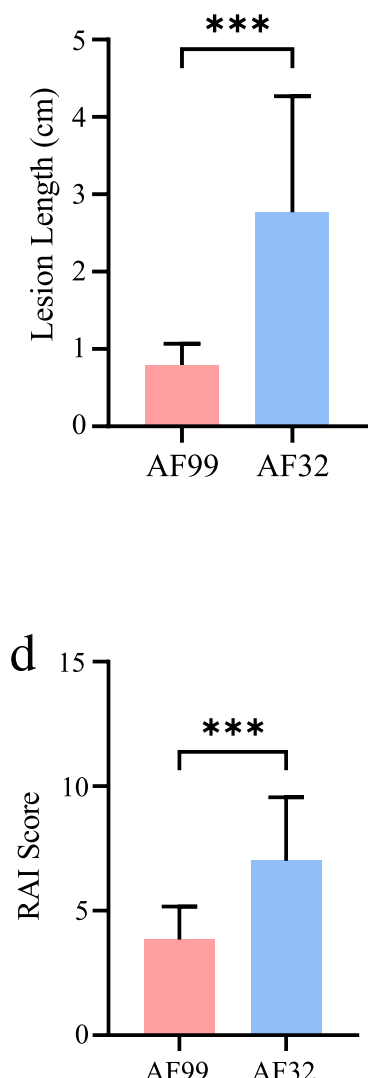

Fig. 1 Phenotypic investigation of two maize lines at pre-harvest and post-harvest times. a. Harvested ears of AF99 and AF32 inoculated by A. flavus spore suspension at pre-harvest time (15 days after pollination). b. Lesion length of ears in AF99 and AF32.Values are means $\pm S E ; ~ n=15 ;$ *** Significant difference by T-test $(P<0.001)$. c. Maize kernels of AF99 and AF32 co-incubated with the fungal plate at post-harvest time. $\mathbf{d}$. RAI score of kernels in AF99 and AF32. RAI score indicates the proportion of hyphae and spores covering the kernel surface. Values are means \pm SE; $n=60$; ** Significant difference by T-test $(P<0.001)$

resulted in a large fungal plaque in AF32 (Fig. 1a). The fungal plaque was significantly longer in AF32 than in AF99 (Fig. 1b). In the post-harvest inoculation, the fungal coverage ratio on the kernel surface (RAI score) of AF32 was significantly higher than that of AF99 (Fig. 1c, d). Hence, these two maize inbred lines vary in resistance to A. flavus and AF99 was more resistant than AF32 both in the pre-harvest and post-harvest inoculations.

\section{Global transcriptome sequencing of the two maize inbred lines}

To investigate the transcriptome dynamics during the early stage of infection by $A$. flavus, we performed RNAseq analysis on the kernels of AF99 (Resistant line) and AF32 (Susceptible line) under A. flavus inoculation (samples designated $\mathrm{R}$ and $\mathrm{S}$ ) and $\mathrm{ddH}_{2} \mathrm{O}$ inoculation (samples designated $\mathrm{RC}$ and $\mathrm{SC}$ ) at each time point (T0, T1, T2, T3, and T4, as described in the Methods). In total, 60 libraries were constructed and sequenced. For each library, about $6.8 \mathrm{~Gb}$ data were generated. After filtering low-quality reads, each library contained more than 45 million reads, $81.17 \%$ of which were mapped to the B73 reference genome (Table S1). The pairwise Pearson's coefficients between the biological replicates were greater than 0.97, indicating a high consistency among the replicates (Figure S1). To further validate the RNAseq data, five genes were randomly selected for real-time RT-PCR analysis (Figure S2, Table S2). The results indicated the reliability of the results of RNA-seq and the data could be used for further analysis.

\section{Identification of DEGs between control and infected maize kernels during infection}

We conducted pairwise comparisons between mockinoculated and fungal-inoculated kernels ( $\mathrm{R}$ vs. $\mathrm{RC}, \mathrm{S}$ vs. $\mathrm{SC})$ to identify genes that respond to A. flavus infection at each time point in each inbred line. At the beginning stage (T0 stage), 395 (R0 vs. RC0) and 300 (S0 vs. SC0) DEGs were identified in AF99 and AF32, respectively (Table 1). These might be the initial genes that involved 
Table 1 The number of up- and down-regulated DEGs at different time points after inoculation

\begin{tabular}{|c|c|c|c|c|c|c|c|c|c|c|}
\hline \multirow{2}{*}{$\begin{array}{l}\text { Paired } \\
\text { samples }\end{array}$} & \multicolumn{2}{|c|}{ Ohpi (T0) } & \multicolumn{2}{|c|}{0.5 hpi (T1) } & \multicolumn{2}{|c|}{ 1.5hpi (T2) } & \multicolumn{2}{|c|}{ 3hpi (T3) } & \multicolumn{2}{|c|}{ 6hpi (T4) } \\
\hline & Up & Down & Up & Down & Up & $\overline{\text { Down }}$ & Up & Down & Up & Down \\
\hline$R$ vs $R C$ & 207 & 188 & 99 & 130 & 377 & 157 & 197 & 192 & 503 & 296 \\
\hline S vs SC & 145 & 155 & 91 & 240 & 306 & 428 & 1243 & 86 & 838 & 1225 \\
\hline RC vs SC & 3350 & 2341 & 2873 & 2650 & 4416 & 1963 & 4970 & 1772 & 3059 & 2615 \\
\hline$R$ vs $S$ & 3206 & 2371 & 3005 & 2624 & 5075 & 1865 & 3438 & 2346 & 4001 & 2392 \\
\hline
\end{tabular}

$R$ and $R C$ The resistant line AF99 was challenged with pathogen (R) or treated with water (RC), $S$ and SC The susceptible line AF32 was challenged with pathogen (S) or treated with water (SC), hpi Hours post inoculation

in the host response. Following the T0 stage, the number of DEGs was constantly changing in both lines (Table 1). These discovered DEGs were differentiated between the two lines at each time point, both in gene number and gene function (Figure S3). Only a few of these DEGs showed similar expression patterns after inoculation with $A$. flavus $(5,2,47,70$, and 204 genes were upregulated in both lines, and 4, 14, 7, 2, and 69 genes were down-regulated in both lines at each time point, respectively) (Figure S3), suggesting that these genes might respond to $A$. flavus infection in both lines. We also analyzed the gene expression patterns in the same genotype during infection, and found that only a few genes were differentially expressed for more than one period (Figure S4, S5). Taken together, the response of maize kernel to $A$. flavus infection was genotype-specific and infection stage-specific.

Based on the GO analysis of the above DEGs, few terms were enriched until the T3 stage, and then numerous genes were preferentially associated with several GO terms (Fig. 2a). At the T4 stage, up-regulated genes in fungal-inoculated AF99 (R) were enriched in several different terms, which mainly related to defense responses, including "response to external biotic stimulus" (GO:0043207, corrected $p$-value $=0.0019$ ), "response to oxidative stress" (GO:0006979, corrected $p$-value $=$ 0.0001), "response to toxic substance" (GO:0009636, corrected $p$-value $=0.0008)$, "reactive oxygen species metabolic process" (GO:0072593, corrected $p$-value $=$ 3.03E-06), "response to fungus" (GO:0009620, corrected $p$-value $=0.045)$, and "hormone binding" (GO:0042562, corrected $p$-value $=0.043)$. We also observed kernel development-related terms based on down-regulated genes, like "seed development" (GO:0048316, corrected $p$-value $=0.0001)$ and "embryo development" (GO: 0009790, corrected $p$-value $=0.0028) \quad($ Fig. $2 \mathrm{a})$. In the susceptible line AF32, up-regulated genes were enriched in relatively few pathways associated with plant defense, while down-regulated genes were also clustered into some immune-related terms, such as "oxidoreductase activity" (GO:0016491, corrected $p$-value $=0.0267$ ), "hormone-mediated signaling pathway" (GO:0009755, corrected $p$-value $=0.0002)$, "response to endogenous stimulus" (GO:0009719, corrected $p$-value $=5.37 \mathrm{E}-06)$, and "induced systemic resistance, jasmonic acid-mediated signaling pathway" (GO:0009864, corrected $p$-value $=$ 0.009) (Fig. 2a). KEGG analysis showed that the synthesis of some metabolites associated with plant resistance, such as benzoxazinoids, steroids, and carotenoids, was inhibited in the susceptible line after inoculation (Figure S6).

Next, we visualized the expression profiles of the DEGs in response to A. flavus at stage T4 via the MapMan tool. According to the general overview of the cellular response, the biotic stress response was the primary response induced (Figure S7). It was obvious that most DEGs (220/260) responding to biotic stress were up-regulated in the AF99, while only half of the genes (344/665) were up-regulated in AF32 (Fig. 2b, Table S3). Nine WRKY genes in AF99 (all up-regulated) and eight (half up-regulated) in AF32 were identified, and three of them (LOC100193498, LOC100501702, and LOC103635353) were commonly up-regulated in both lines. Most genes encoding other transcription factors, such as ERF, bZIP, MYB, and DOF, were down-regulated in the susceptible line, as well as hormone-related genes. Two-thirds of genes in the ethylene pathway were upregulated in the resistant line AF99. Among them, LOC103651133, encoding ethylene response sensor 1, was up-regulated (13.9-fold). In the susceptible line AF32, half of the ethylene pathway genes were down-regulated, and the top two in the fold-change ranking were the DEAD box RNA helicase pseudogene (LOC100272753, 19.6-fold) and the ethylene-responsive transcription factor ERF109like (LOC103647485, 16-fold). In addition, most of other genes that participate in defense pathways, like cell wall, signaling, and proteolysis, were up-regulated in the resistance line and down-regulated in the susceptible line.

\section{Identification of DEGs between the two maize inbred lines during infection}

With or without challenge by A. flavus, more than 5000 genes at each stage were identified to be differentially expressed between the two lines ( $\mathrm{RC}$ vs. SC, R vs. S), suggesting that the expression of these genes was 


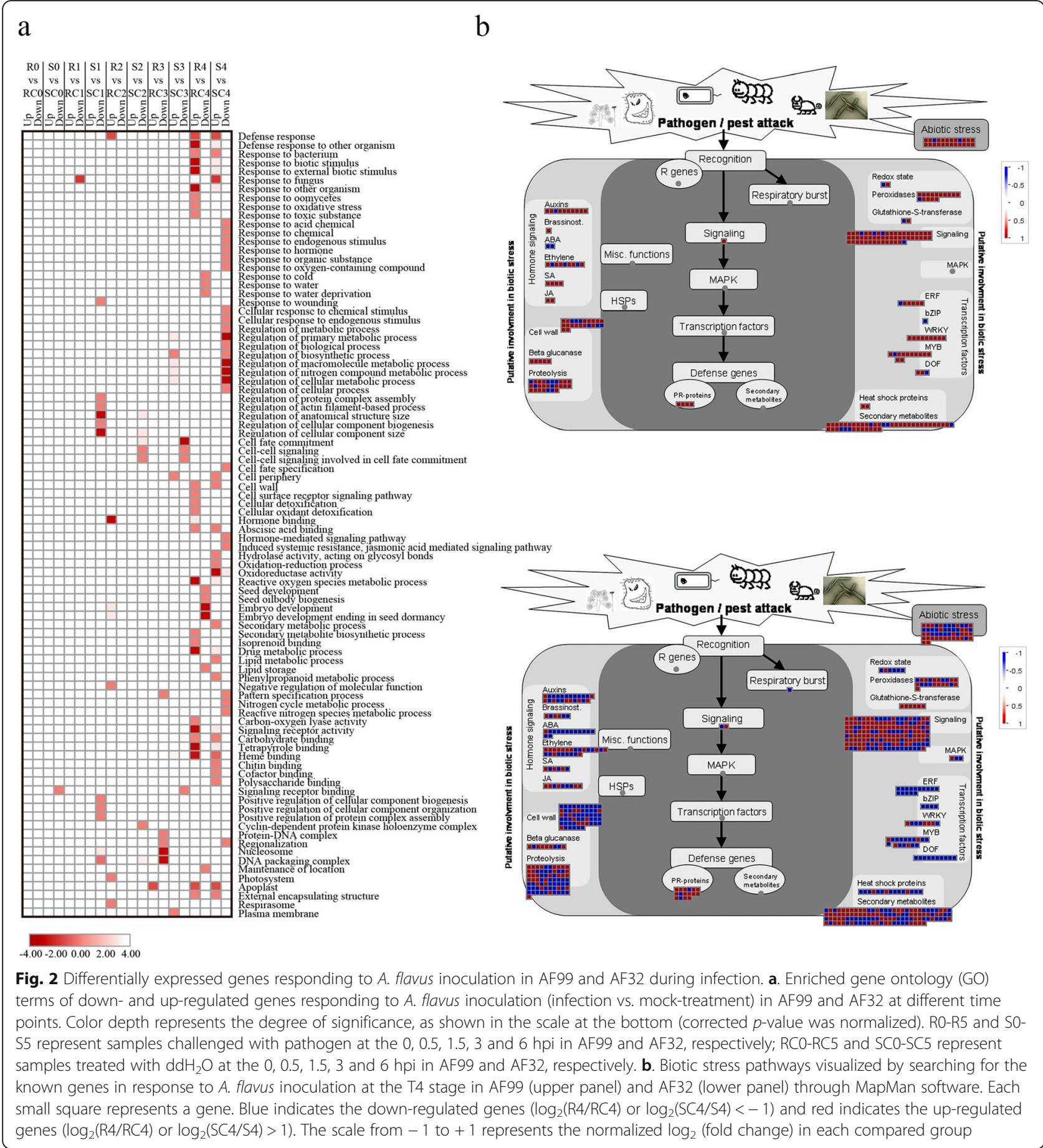

influenced by their genetic backgrounds (Table 1). Venn diagrams revealed that a small subset of the DEGs showed differential expression only in fungal-inoculated kernels (R vs. S) (Fig. 3a), indicating that their expression difference might be induced by fungal infection. Taking the T0 stage for example, 2353 and 1573 DEGs were commonly up-regulated or down-regulated, regardless if it was inoculated with A. flavus. But 851 and 788 DEGs were up-regulated or down-regulated only in the fungalinoculated kernels, respectively (Fig. 3a).

Next, we investigated the DEGs only in the fungalinoculated kernels at the five infection stages through functional enrichment analysis. According to the GO analysis, we discovered that these up-regulated DEGs are mostly involved in plant stress responses, such as "response to drug" (GO:0042493), "response to antibiotic" (GO:0046 

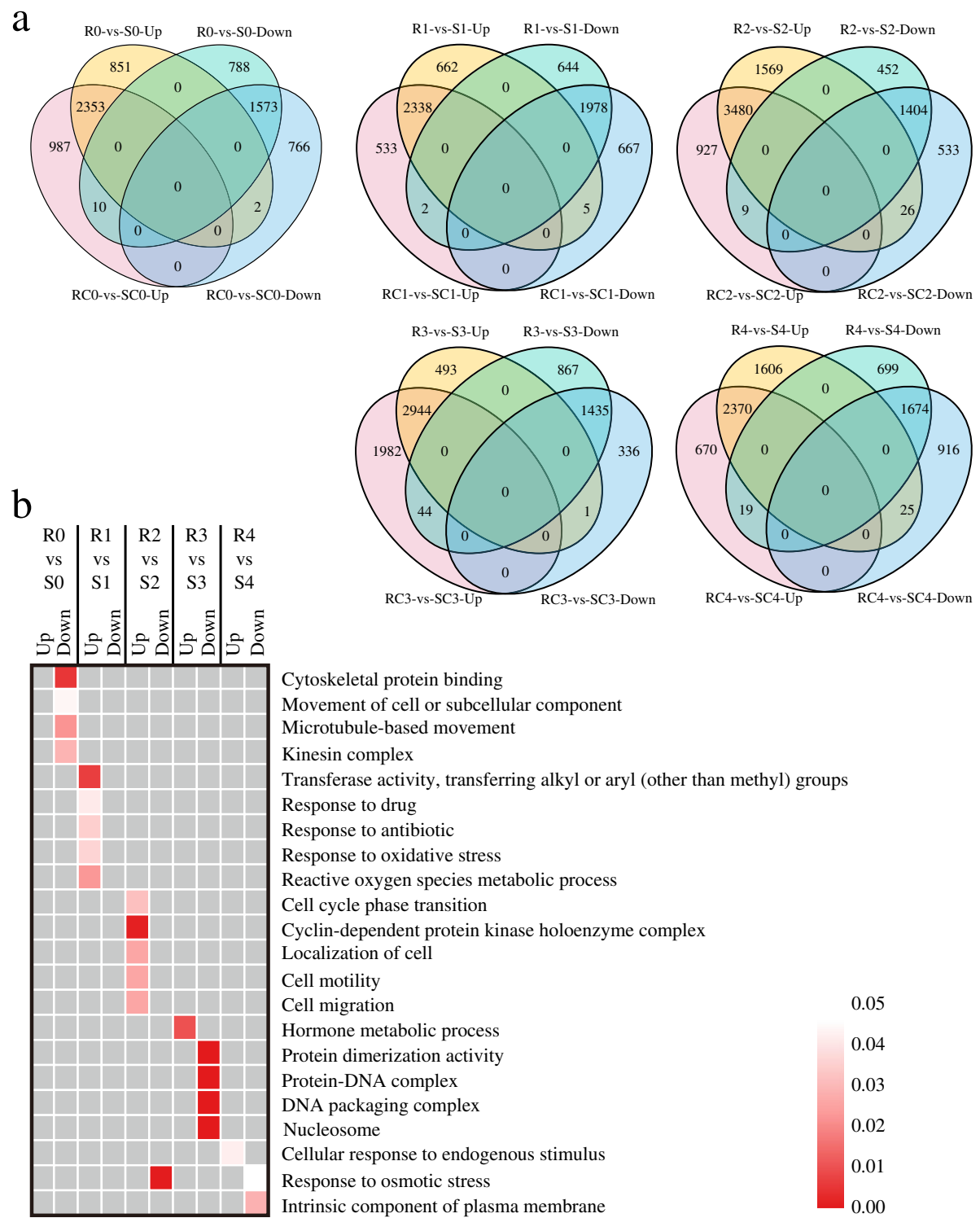

Fig. 3 Differentially expressed genes between AF99 and AF32 with or without challenged by A. flavus. a. Venn diagrams showing the number of DEGs between AF99 and AF32 at each stage of A. flavus inoculation. A total number of up- and down-regulated genes (log fold change $\geq 1$ or $\leq-1$ and $P<0.05$ ) in AF99 as compared to AF32 in mock-inoculated (RC vs. SC) and fungal-inoculated (R vs. S) groups. b. Enriched gene ontology terms in the specific differentially expressed genes of fungal-inoculated kernels between AF99 and AF32. Color depth represents the degree of significance, as shown in the scale at the right (corrected $p$-value)

677), "reactive oxygen species metabolic process" (GO: 0072593), and "hormone metabolic process" (GO:0042445) (Fig. 3b). The KEGG analysis also suggested that these upregulated genes were involved in different signaling pathways and biosynthesis of secondary metabolites, such as antibiotics and prodigiosin (Figure S8), whereas the down-regulated DEGs were not enriched in the defense response. These results indicate that those specific upregulated DEGs in fungal-inoculated kernels might be involved in the immune response upon A. flavus challenge.

\section{Gene co-expression analysis during A. flavus infection by WGCNA}

To facilitate our understanding of the regulatory network of the maize genotype-specific and infection stagespecific response to A. flavus infection, 60 samples and their expression data sets, including 34,315 genes, were subjected to WGCNA. Then, 15,409 genes (the first 75\% of the median absolute deviation (MAD), MAD>0.25) were selected to construct a directed network. We chose a power of $\beta=12$ based on the scale-free topology 


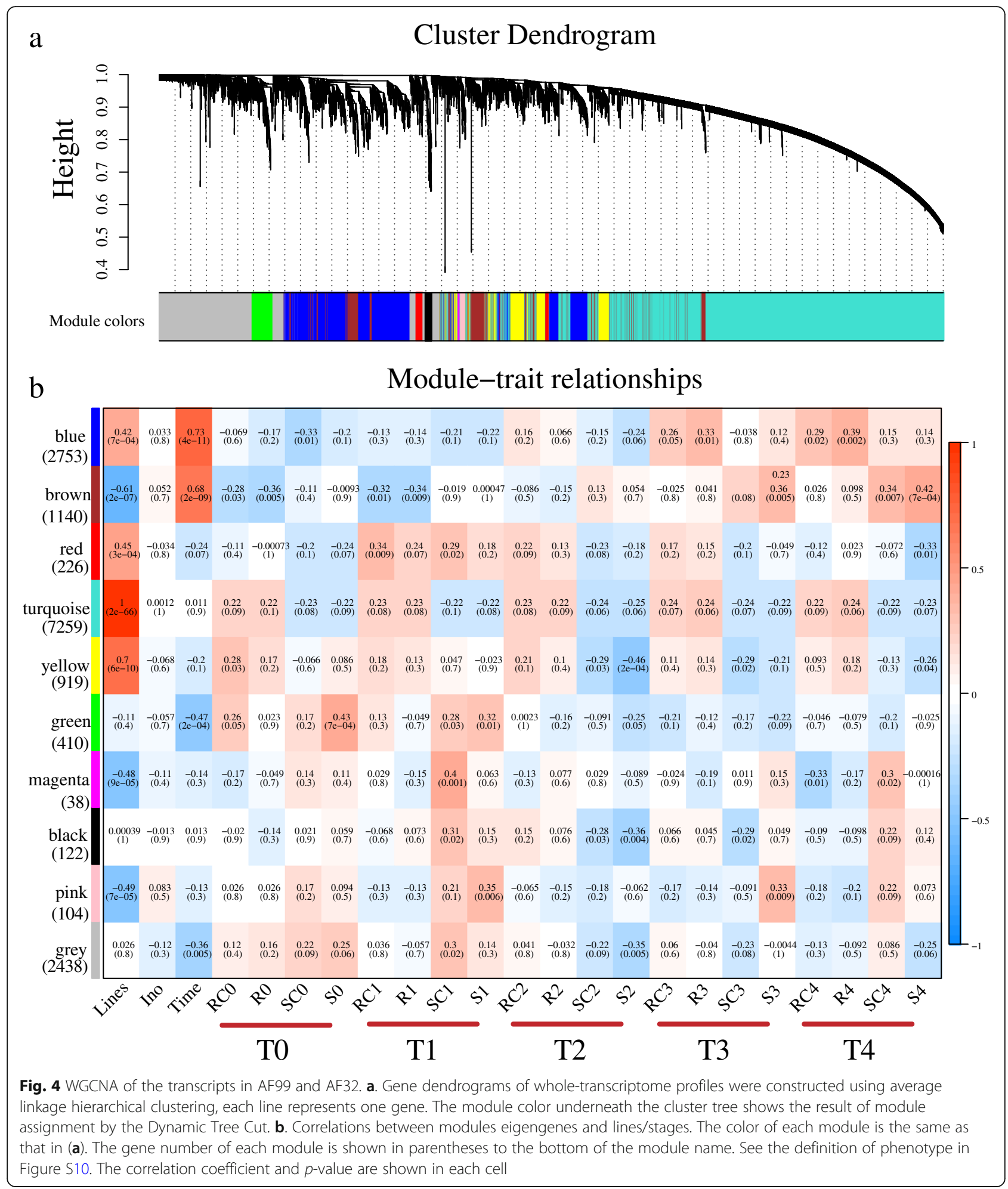

criterion to generate a hierarchical clustering tree (Figure S9, Fig. 4a). A total of 9 co-expression modules (mergeCutHeight $=0.25$ ) were ultimately identified, with the gene number of each module ranging from 38 to 7259. Every module was marked with different colors, and 2438 genes that did not belong to any modules were put into the grey module.

Then the correlations between the modules' eigengenes and genotypes/stages were studied (Figure S10, Fig. 4b). Most of the modules obviously correlated with 
the different maize inbred lines, of which four modules (blue, red, turquoise, and yellow) showed significantly positive correlations, and three modules (brown, magenta, and pink) showed significantly negative correlation. The turquoise module, which consists of nearly half of all the genes used in the WGCNA, showed extremely high correlation to the maize inbred lines $(r=1$, $p=2 \mathrm{e}-66$ ). Notably, the expression of these genes in the turquoise module was almost impervious to fungal inoculation or infection stages (Fig. 4b), implying that they might not be involved in the resistance to A. flavus in the early stage.

We found that the gene expression patterns in three modules (blue, brown, and green) were significantly correlated with infection time. For instance, the expression levels of genes in the blue and brown modules were low before the T2 stage, and then gradually increased in stage T3 and T4 to various extents, while genes in the green module showed the opposite pattern. Nevertheless, no modules was identified to be associated with inoculation (mock-inoculated or fungal-inoculated, ino), which was probably due to the tremendous differences in their genetic background and rapid and dynamic changes in gene expression.

To investigate the precise gene regulatory network during infection, we performed WGCNA by connecting gene co-expression modules to the four groups $(\mathrm{R}, \mathrm{RC}$, $\mathrm{S}, \mathrm{SC})$ in the successive infection stages. In general, 7 modules (blue, brown, green, yellow, black, pink, and magenta) were found to be associated with specific infection stages (Fig. 4b). At the T0 stage, the green module (410 genes) was positively correlated with S0 $(r=0.43, p=7 \mathrm{e}-04)$, while the brown module (1140 genes) had a markedly negative relationship with R0 ( $r=$ $-0.36, p=0.005)$. At the T1 stage, the magenta module (38 genes) and the pink module (104 genes) were positively correlated with $\mathrm{SC} 1(r=0.43, p=0.001)$ and $\mathrm{S} 1$ $(r=0.35, p=0.005)$, respectively. At the T2 stage, both the yellow module (919 genes) and the black module (122 genes) had significant negative correlations with S2. At the T3 and T4 stages, the brown module was positively correlated with S3 $(r=0.36, p=0.005)$ and S4 $(r=0.44, p=7 \mathrm{e}-04)$, while the blue module (2753 genes) was positively correlated with R4 $(r=0.39, p=0.002)$ (Fig. 4b). The significant correlations between stagespecific gene expression and module membership (MM) in each module were shown (Fig. 5a, d, Fig. 6a, d, and S11). Four key modules (gene number $>400$ ) were further analyzed in the following sections.

\section{Characteristics and hub genes of the transcriptional} regulatory modules correlated with different infection stages In the green module, genes were up-regulated in fungalinoculated AF32 (S) at the T0 stage compared with mock-inoculated group (Fig. 5b). According to the GO analysis, we obtained 50 significant GO terms (corrected $p<0.05$ ), and the top two terms were "plasma membrane protein complex" (GO:0098797, corrected $p$-value $=2.36 \mathrm{E}-08$ ) and "cell cycle process" (GO:0022402, corrected $p$-value $=5.04 \mathrm{E}-07$ ) (Fig. $5 \mathrm{c}$, Table S4). KEGG analysis indicated that these genes are closely related to RNA transport (Fig. 5g). In addition, 13 hub genes were identified (Fig. 5i, Table S8), including LOC109939959 encoding a homolog of the Arabidopsis thaliana DNA demethylase REPRESSOR OF SILENCING 1 (ROS1, AT2G36490). The Arabidopsis ros1 mutant shows higher methylation levels of many gene promoters and increased susceptibility to pathogens [33-36]. Another hub gene, LOC103631592, might also be involved in regulating methylation levels, because its Arabidopsis homolog AT5G04290 (SPT5L) is required for RNAdirected DNA methylation [37, 38]. Consistently, GO analysis also indicated that genes in the green module were enriched in "demethylation" (GO:0070988, corrected $p$-value $=0.0019)$, "gene silencing by RNA" (GO: 0031047 , corrected $p$-value $=0.0184$ ), and "gene silencing" (GO:0016458, corrected $p$-value $=0.0192$ ). Collectively, we speculated that the change of the genome methylation level and gene silencing might be related to the maize kernel resistance in the early stage of $A$. flavus infection.

In the yellow module, the expression level of genes was drastically reduced at the $\mathrm{T} 2$ stage in the $\mathrm{S}$ and $\mathrm{SC}$ groups, and the decrease in $\mathrm{S} 2$ was more pronounced than that in SC2 (Fig. 5e). GO enrichment analysis revealed that 12 GO terms were significantly over-represented (corrected $p$-value <0.05), including "small molecule metabolic process" (GO:0044281, corrected $p$-value $=1.94 \mathrm{E}-10)$ and "cellular response to endogenous stimulus" (GO:0071495, corrected $p$-value $=0.0450) \quad($ Fig. $5 f$, Table S5). The top two pathways with the highest significance were "biosynthesis of antibiotics" and "fatty acid biosynthesis" based on KEGG pathway analysis (Fig. 5h). Additionally, a total of 23 hub genes in this module were screened out. Five (fab1, LOC100192829, LOC100281497, gpm519, and fae1) and two (pco065117 and LOC100191601) of these hub genes are directly related to fatty acid biosynthesis and lipid metabolic processes, respectively (Fig. 5j, Table S8).

In the brown module, the expression of these genes increased in the SC and S groups, and the increase in S2 was more evident (Fig. 6b). According to GO and KEGG analysis, only six terms (corrected $p$-value $<0.05$ ) and three enriched pathways $(Q$ value $<0.05)$ were identified (Fig. 6c, g, Table S6). Of the 19 hub genes identified in this module (Fig. 6i, Table S8), three Barwin family proteins (LOC100282340, LOC103652813, and LOC1036 52814 ) with chitinase activity might play vital roles as PR proteins in restriction of $A$. flavus infection $[39,40]$. 


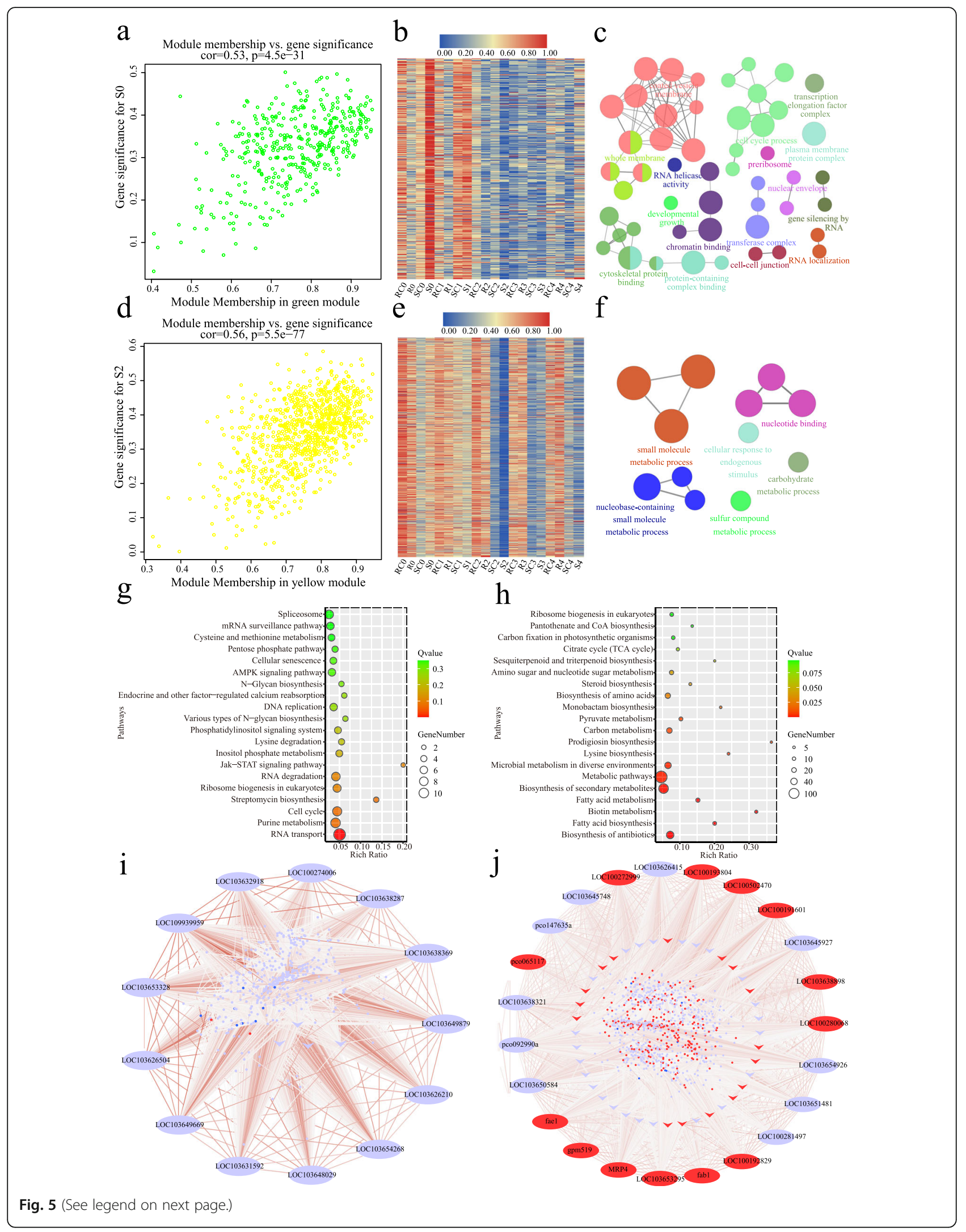


(See figure on previous page.)

Fig. 5 Expression profile and transcriptional regulatory network in the green and yellow modules. a, d Scatterplots of gene significance versus module membership for the green/S0 (a) and yellow module/S2 (d), with correlations and $p$-value indicated. $\mathbf{b}$, e Heat map of genes in the green (b) and yellow module (e). Red indicates high expression, blue indicates low expression. The color scale represents Z-score. c, $\mathbf{f} \mathrm{GO}$ analysis of genes in the green $(\mathbf{c})$ and yellow module $(\mathbf{f})$. Each circle represents an enrichment category, and the size of the circle indicates the number of genes. Detailed enrichment results are shown in Table S4 and S5. g, h Top 20 associated KEGG pathways for the green (g) and yellow module (h). $\mathbf{i}, \mathbf{j}$ Coexpression network of the green module (i) and yellow module (j). The ellipses of the outer circle represent the hub genes, the arrows in the inner circle represent transcription factors, and the small dots in the circles represent other coexpressed genes in each module. The relationships of all the genes are connected by lines, the line color represents the weight. The red ellipse /arrow/dot indicates up-regulated $\left(\log _{2}(R / S)>1\right)$ and the blue ellipse /arrow/dot indicates down-regulated in the resistance line $\left(\log _{2}(R / S)<-1\right)$ at two spectific stages (T0 for the green module and T2 for the yellow module)

$L O C 100193776$, as the ortholog of $L A Z Y 1$ in rice, participates in regulating auxin transport and auxin signaling, was also a hub gene of the brown module [41]. In addition, four other hub genes (LOC100382449, pco080661a, LOC100273283, and LOC103632841) are considered to be involved in the response to various biotic or abiotic stresses.

In the blue module, the expression of genes increased in the $\mathrm{RC}$ and $\mathrm{R}$ groups, and the trends were more obvious after A. flavus infection (R) (Fig. 6e). According to GO and KEGG analysis, there were 71 terms and 32 pathways with statistical significance (Fig. 6f, h, Table S7). Remarkably, in the blue module, 226 genes were involved in "oxidation-reduction process" (GO:0055114, corrected $p$-value $=0.0017$ ) and 8 genes were associated with "plant-type hypersensitive response" (GO:0009626, corrected $p$-value $=0.0075$ ) (Fig. 6f, h). KEGG analyses also showed that genes in this module were enriched in pathways such as "biosynthesis of antibiotics", "biosynthesis of amino acids", "glycolysis/gluconeogenesis", and "fatty acid degradation" (Fig. 6g). Totally 43 hub genes in this module were identified in this module (Fig. 6j, Table S8). Six (LOC103636223, LOC100382469, LOC10 3650090, LOC100284999, LOC103626647, and pco10 2102) of them are closely related to the biosynthesis of antibiotics. Another hub gene, IDP2565 encoding aPR10 protein, is vital in maize host defense, and PR10 RNAisilenced mature kernels showed more fungal colonization and aflatoxin production $[17,42]$. The hub gene LOC100274556 is a homolog of AT1G68010, whose protein possesses catalase activity and confers tolerance to multiple abiotic stresses in Arabidopsis [43, 44].

Taken together, a total of 110 hub genes in the 7 modules correlated with different infection stages. These genes were enriched in the GO terms, "cellular amine metabolic process", "defense response to fungus", and "fatty acid synthase activity" (Figure S12, Table S9). The top two KEGG pathways were "fatty acid biosynthesis" and "biosynthesis of antibiotics" (Figure S12). Hence, these hub genes and biological pathways might play an important role in modulating the defense response to A. flavus infection in maize.

\section{Integration of previously identified candidate genes and transcriptome data}

A list of 195 candidate genes for maize resistance to A. flavus infection and/or aflatoxin contamination has been reported [45]. Interestingly, we noticed differential expression of these genes in both lines in the early stage of infection. In the first $6 \mathrm{hpi}, 90$ of these genes were found to be differentially expressed (Fig. 7). The expression differences between genotypes (RC vs. SC, R vs. S) were significantly higher than those between treatments ( $\mathrm{RC}$ vs. $\mathrm{R}, \mathrm{SC}$ vs. S). Compared with the control groups (RC and SC), 14 and 42 genes were differentially expressed in AF99 and AF32, respectively. Eight of these genes (LOC103652813, LOC103639781, LOC10028 0605, TIDP2793, LOC100285638, LOC100384012, LOC1003 84000 , and LOC103633275) were induced in both lines. At the T4 stage, 6 genes were up-regulated in AF99, including Barwin-like (LOC103652813), the transcription factor MYB41 (LOC100037746), chitinase 2 (LOC100285638), WRKY transcription factor 23 (LOC103654285), probable WRKY transcription factor 53 (LOC103639781), and LOC100272820 encoding an unknown protein. Among them, LOC103652813, LOC100285638, LOC100272820, and LOC103639781 were also up-regulated in AF32. In addition, 18 genes were down-regulated in AF32, while only 2 genes were down-regulated in AF99, indicating that more diseaseresistance were induced and then contributed to the resistance of AF99.

We also analyzed the relationship between these candidate genes and our hub genes obtained by WGCNA, and found that 3 of the 110 hub genes were in the list. Two hub genes in the blue module, LOC100279513 and LOC100285591, were either linked to QTLs in two mapping populations or associated with aflatoxin accumulation in the previously reported GWAS panel [45]. One hub gene (LOC103652813) of the green module, located in bin 4.02, was also in the consolidated list [45].

\section{Discussion}

The molecular mechanisms and precise regulatory network underlying the defense system of maize kernel in response to A. flavus in the early stage are limited. In this work, we focused on the transcriptome reprogramming of resistant and susceptible lines within $6 \mathrm{~h}$ of fungal 


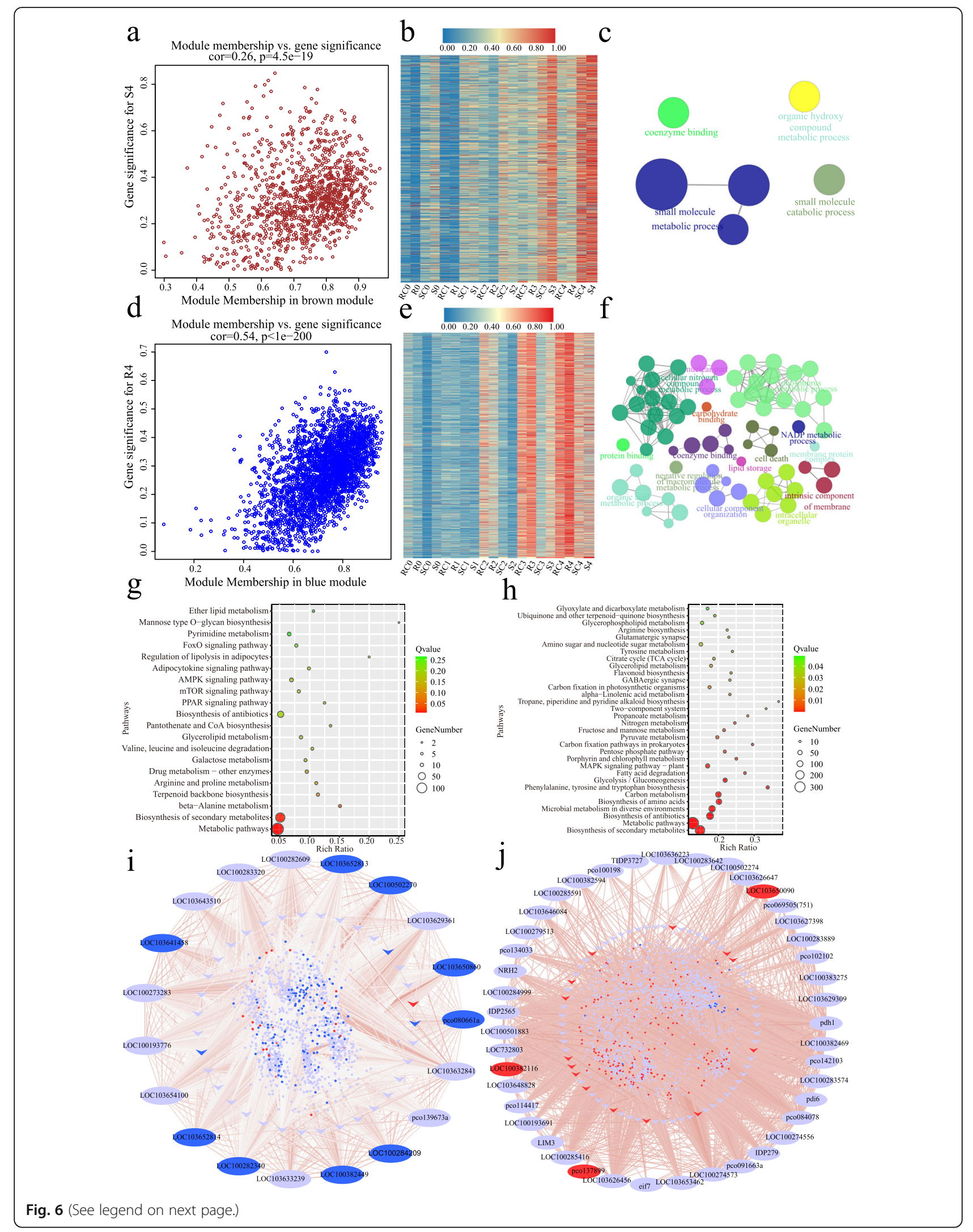


(See figure on previous page.)

Fig. 6 Expression profile and transcriptional regulatory network in the brown and blue modules. a, $\mathbf{d}$ Scatterplots of gene significance versus module membership for the brown/S4 (a) and blue module/R4 (d), with correlations and p-values indicated. $\mathbf{b}$, e Heat map of genes in the brown (b) and blue module (e). Red indicates high expression, blue indicates low expression. The color scale represents Z-score. c, $\mathbf{f} \mathrm{GO}$ analysis of genes in the brown (c) and blue module (f). Each circle represents an enrichment category, and the size of the circle indicates the number of genes. Detailed enrichment results are shown in Table S6 and S7. g, h KEGG analysis of genes in the brown (g, top 20) and blue module (h, corrected $p$-value $<0.05)$. $\mathbf{i}, \mathbf{j}$ Coexpression network of the brown module (i) and blue module (j). The ellipses of the outer circle represent the hub genes, the arrows in the inner circle represent transcription factors, and the small dots in the circles represent other coexpressed genes in each module. The relationships of all the genes are connected by lines, the line color represents the weight. The red ellipse /arrow/dot indicates up-regulated $(\log 2(R / S)>1)$ and the blue ellipse /arrow/dot indicates down-regulated in the resistance $\operatorname{line}(\log 2(R / S)<-1)$ at the T4 stage

infection. DEGs induced by A. flavus infection or between the two lines were analyzed. More than 300 DEGs were identified in both lines at the T0 stage (Table 1), while only one genes were found at the early infection stage in a previous study [29], which reveals that the response of maize kernels to fungal invasion is very rapid. This difference might be due to the improved fungi inoculation method employed in our study. Here, maize kernels were cut longitudinally and immersed into the spore suspension. The full contact between maize cells and A. flavus may eliminate the interference caused by the seed coat. The kernels inoculated at $0,0.5,1.5,3,6$ hpi were sampled in this study and DEGs varied at each time point, which means a dynamic regulation occur in the early stage. The density of our sampling time will facilitate our understanding of the precise expression regulation network of maize against $A$. flavus in the initial stages. Compared with mock-inoculated groups, the susceptible line AF32 had more DEGs than the resistant line AF99, except for at the T0 stage (Table 1). This finding is in agreement with the previous results that 214 and 2159 genes were induced in resistant and susceptible kernels at $72 \mathrm{hpi}$, respectively [46]. These results suggest that the susceptible lines are vulnerable to $A$. flavus infection, leading to a dramatic change in gene expression after inoculation.

Based on the dynamic transcriptome analysis, A. flavus infection induced a large number of DEGs in both lines, most of which are related to host defense (Fig. 2). Among them, $84 \%$ of DEGs were up-regulated after inoculation in AF99(R), while only $48 \%$ were up-regulated in AF32. (Fig. 2). Besides, a majority of 90 candidate resistance genes showed a higher basic expression level in AF99 than in AF32 (Fig. 7). Taken together, these might be the leading cause of the difference in resistance (Fig. 2).

Integrated GO analysis, KEGG analysis and WGCNA demonstrated that $A$. flavus resistance in maize is genotype-/ stage-specific (Figs. 2, 3, 4, 5, 6). WGCNA is a well-established tool in systems biology, which can be used to describe correlation patterns among genes across multiple samples. Through WGCNA, we adopted the whole-genome expression data to construct complete co-expression networks, and 7 modules were considered to be correlated with different infection stages (Fig. 4). GO and KEGG analysis in these modules revealed that the genes participated in plasma membrane protein complex, cell cycle process, RNA transport, and DNA methylation were affected in the initial stage of the infection (T0 and T1); some secondary metabolic processes, like biosynthesis of antibiotics, fatty acid biosynthesis, and biotin metabolites, were activated in response to infection at the T2 stage; more DEGs or hub genes involved in hormone signaling, antibiotic and fatty acid biosynthesis, lipid storage, and hypersensitive cell death responses (HRs) were discovered at stage $\mathrm{T} 3$ and $\mathrm{T} 4$ (Figs. 3, 4, 5, 6, S6, S8). Therefore, our WGCNA provided new genetic resources for molecular breeding and deepened our understanding of maize resistance to $A$. flavus.

A. flavus is a necrotrophic fungal pathogen that feeds on dead host cells. Traditionally, it is believed that the $\mathrm{HR}$, as well as HR-triggered programmed cell death (PCD) can enhance necrotrophic pathogen virulence [47-49]. However, in recent years, some studies have reported that increasing the expression of some genes that trigger the HR may also enhance plant resistance to necrotrophic pathogens [50, 51]. Herein, functional analysis of hub genes and GO enrichment analysis of the 7 modules correlated with infection stages revealed that genes involved in HR and PCD might play a crucial role in maize resistance against $A$. flavus (Figs. 5, 6, 7, Table S8). We analyzed 35 genes related to the HR and PCD and found 11 were in the blue module and up-regulated in $\mathrm{AF} 99(\mathrm{R})$ at the T4 stage (Figure S13). Among them, LOC100502274 is a homolog of AT4G38360 (LAZ1) who contributes to PCD associated with the HR in Arabidopsis [52, 53]. However, 4 of the 35 genes were upregulated at the T4 stage in AF32(S), and 3 of which were clustered in the brown module (Figure S13). In particular, two genes (LOC103652814 and pco080661a) are the hub genes of the brown module, implying that HRs were stimulated at the T4 stage in the susceptible line. These results indicated that PCD and HRs might be triggered after A. flavus inoculation in both lines, and further experiments are needed to verify their relationship with maize host defense against $A$. flavus. 


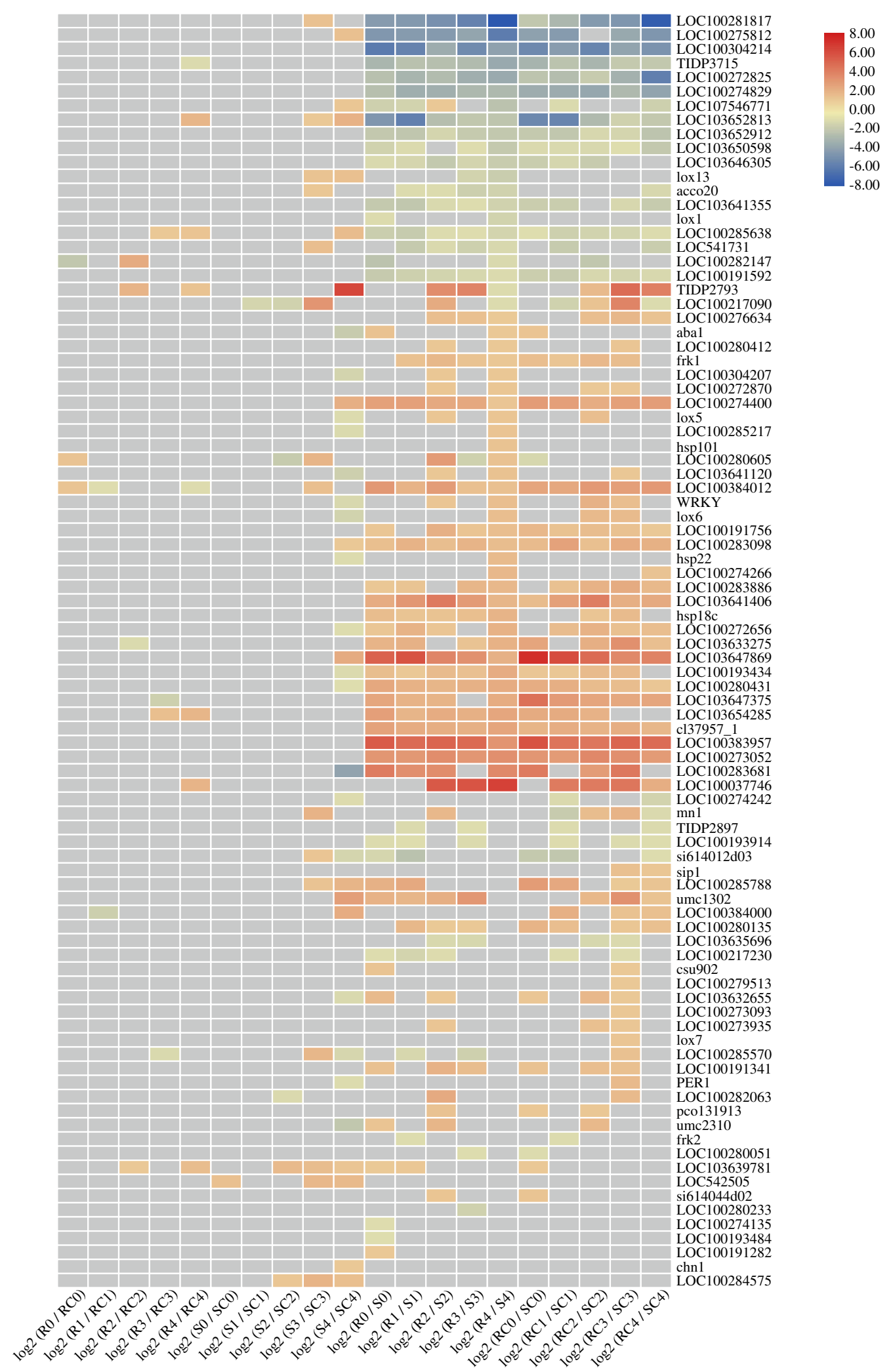

Fig. 7 Heat map of 90 candidate genes for maize resistance to A. flavus infection and/or aflatoxin contamination. Red indicates up-regulated, blue indicates down-regulated. The gray cells indicate no differential expression. The color scale represents Z-score 
The KEGG enrichment analyses suggested that genes related to antibiotics synthesis might be involved in regulating host defense (Figs. 3, 5, 6, S8, S12). Plantderived antibiotics are antimicrobial secondary metabolites produced by the plant. They contain both preformed antifungal compounds constitutively present in healthy plants and induced antifungal compounds (phytotoxin) synthesized in response to pathogen invasion [54]. In maize, dynamic regulation of several antibiotics, including benzoxazinoids, phenylpropanoids, oxylipins, and terpenoids, contributes greatly to host resistance when challenged with diverse pathogens [55-61]. In our study, genes regulating phenylpropanoid, diterpenoid, and terpenoid backbone biosynthesis were up-regulated in response to fungus attack in both the resistant and susceptible lines (Figure S6). Based on the results of WGCNA, 58 and 388 genes involved in biosynthesis of antibiotics were enhanced in AF99 in the yellow and blue modules, respectively; while only 38 related genes were found in AF32 in the brown module. These results suggested that AF99 might have a greater ability to synthesize antibiotics (Figs. 5, 6). Therefore, we speculated that plant-derived antibiotics participate in the regulation of maize resistance to A. flavus in the early stage of infection and might be an important factor underlying resistance variation among genotypes.

\section{Conclusions}

Collectively, RNA-seq data generated from two maize lines with contrasting resistance in the pre-harvest and post-harvest inoculation provided a robust resource to study maize kernel resistance to A. flavus. Herein, we investigated the genotype-specific and infection stagespecific response of maize against $A$. flavus infection, and found that inhibition of some defense pathways might lead to the reduced host resistance in the susceptible line. We also constructed gene co-expression networks during the first 6 hpi by WGCNA, and discovered that DNA methylation, biosynthesis of antibiotics, fatty acid biosynthesis, hormone signaling, and HRs greatly influenced host resistance. Further, the 110 hub genes identified in this work could be important targets for maize resistance against $A$. flavus in future breeding efforts.

\section{Methods}

\section{Plant materials and growth conditions}

Two maize inbred lines, AF99 (resistant line) and AF32 (susceptible line), were selected from recombinant inbred lines derived from a cross between RA and Z58. The parental line RA was obtained from a cross between two Chinese elite inbred lines, Ye478 and Dan340, which showed excellent resistance to Aspergillus flavus in years of repeated experiments [62]. The other parental line, Z58, is a Chinese elite inbred line.
All the individuals were planted in the fields at the Experimental Station of Yangzhou University $\left(18^{\circ} 18^{\prime} 06^{\prime \prime} \mathrm{N}\right.$ $\left.109^{\circ} 39^{\prime} 32^{\prime \prime} \mathrm{E}\right)$ during the 2019 growing season. The plants were grown in $0.55 \times 0.25 \mathrm{~m}$ plots, and each plant was self-pollinated to ensure enough materials for further investigation.

\section{Inoculation method and phenotypic evaluation}

The A. flavus strain used in this study was isolated by Professor Yin Shixue (College of Environmental Science and Engineering of Yangzhou University) [63]. A. flavus was grown on modified Czapek agar medium at $30^{\circ} \mathrm{C}$ under dark cultivation for 7 days, and a conidial suspension $\left(2 \times 10^{6}\right.$ spores $\left.\mathrm{mL}^{-1}\right)$ was prepared with sterile water before inoculation.

For the pre-harvest inoculation, maize ears at 15 days after pollination were selected and artificially inoculated with the nail punch method. Two injections per ear were performed with $1 \mathrm{ml}$ of spores per injection. The disease resistance phenotype was identified at harvest by measuring the extent of $A$. flavus growth on the ears.

For the post-harvest inoculation, the harvested ears were inoculated with a conidial suspension in the laboratory. The inoculation procedure and the scoring for kernel resistance to $A$. flavus infection (RAI) were conducted according to the procedure described in our previous study [11, 64]. The RAI scores were divided into 11 grades according to the proportion of hyphae and spores covering the maize kernel surface ( 0 for no infection and 10 for complete infection), and each level represents $10 \%$ coverage.

\section{RNA-seq and DEG analysis}

Well-developed maize ears of AF99 and AF32 were selected at $15 \mathrm{~d}$ after pollination and kernels in the middle of ears were collected for further study. Each kernel was cut longitudinally with a sterile scalpel and divided into two equal parts; half of the kernels were immersed in a spore suspension for $5 \mathrm{~min}$, and the other half were immersed in sterile water for $5 \mathrm{~min}$, recorded as $0 \mathrm{~h}$. Then, all kernel halves were transferred to modified Czapek agar medium and cultured in an incubator at $30^{\circ} \mathrm{C}$. At least four kernel halves were collected as one sample at $0,0.5$, 1.5 , 3, and $6 \mathrm{~h}$ (named T0, T1, T2, T3, and T4, respectively), and rapidly cooled with liquid nitrogen and stored in a deep freezer at $-80^{\circ} \mathrm{C}$. Each sample had three biological repetitions to minimize experimental error. The detailed treatment method was described previously [31].

The total RNA was isolated with the ethanol precipitation protocol and CTAB-PBIOZOL reagent (Vazyme Biotech). RNA was qualified and quantified using a NanoDrop spectrophotometer and an Agilent 2100 bioanalyzer (Thermo Fisher Scientific, MA, USA). 
Transcriptome sequencing was performed on the BGISEQ500 platform (BGI-Shenzhen, China). The raw reads containing the sequencing adapter or with over $5 \%$ unknown bases (' $\mathrm{N}$ ' base) and those whose low-quality reads base ratio (base quality $\leq 5$ ) was greater than $20 \%$ were filtered by SOAPnuke (v1.5.2) [65]. Then the HISA T2 (v2.0.4) [66] was applied to align the clean reads to the maize reference genome GCF_000005005.2_B73_RefGen_v4(https://www.ncbi.nlm.nih.gov/assembly/GCF_ 000005005.2).

RSEM (v1.2.12) [67] was used to calculate the fragments per kilobase of transcript per million mapped reads (FPKM). DESeq2(v1.4.5) [68] was used to identify the DEGs with the criterion of fold change $\geq 2.00$ and adjusted $p$-value $\leq 0.05$.

\section{Real-time RT-PCR}

cDNA was synthesized from $0.5 \mu \mathrm{g}$ of total RNA with HiScript ${ }^{\circ} \mathrm{Q}$ RT SuperMix for qPCR (Vazyme, China) according to the protocol. Quantitative RT-PCR was conducted using the StepOnePlus Real Time PCR system with ChamQ SYBR qPCR Master Mix (Vazyme, China). Gene specific primers (Table S2) were designed with QuantPrime qPCR primer design tool (https:// quantprime.mpimp-golm.mpg.de). The primers ZmUBQ$\mathrm{qRT}+/$ - were used to amplify the ubiquitin 1 as control, and the relative gene expression data was calculated using $2^{-\Delta \Delta \mathrm{Ct}}$ method. Each sample had three biological repetitions with three technical replicates to minimize experimental error.

\section{Gene ontology and KEGG pathway enrichment analysis}

ClueGO (v. 2.5.7) [69], a Cytoscape plug-in software of Cytoscape, was employed to conduct the gene ontology (GO) enrichment analysis ( $p$-value corrected with Bonferroni step down $\leq 0.05$ ). KEGG (https://www.kegg.jp/) enrichment analysis was performed by Phyper (https://en. wikipedia.org/wiki/Hypergeometric_distribution) based on the hypergeometric test ( $p$-value corrected with Bonferroni step down $\leq 0.05$ ). MAPMAN software [70] was used to identify the genes in AF99 and AF32 that responded to A. flavus infection.

\section{Weighted gene co-expression network analysis}

The R package for weighted gene co-expression network analysis (WGCNA) was used for describing correlation patterns among genes across multiple samples [71, 72]. All gene expression data were standardized based on $\log _{2}(1+$ FPKM $)$ values, and soft threshold $=12$, based on the scale-free topology criterion, was selected to generate an adjacent matrix. Then the adjacency matrix was converted to a topological overlap matrix (TOM), and the genes were hierarchically clustered based on dissimilarity between genes. The dynamic tree-cutting algorithm was used to cut the hierarchal clustering dendrogram (mergeCutHeight $=0.25$ ) and modules were defined (the minimum number of modules was 30). To estimate the association of modules with gene-specific expression (genotype-specific or infection stage-specific expression), the binary indicator (stages/genotypes $=1$ and all other samples $=0$ ) was used as described [72, 73]. At each time point, stage-/genotype-specific modules $(|\mathrm{r}|>0.35, p<$ 0.05 ) and hub genes (|gene significance $\mid>0.4$ and |intramodular connectivity in interesting modules $\mid>0.9$ ) were identified. Cytoscape software version 3.6.0 [74] was used to visualize co-expression networks. The Venn diagrams and heatmaps were drawn using the TBtools [75].

\section{Abbreviations}

A. flavus: Aspergillus flavus; hpi: Hours post inoculation; DEG: Differentially expressed genes; WGCNA: Weighted gene co-expression network analysis; QTL: Quantitative trait locus; GWAS: Genome-wide association studies; PCD: Programmed cell death; HR: Hypersensitive cell death response; MAD: Median absolute deviation

\section{Supplementary Information}

The online version contains supplementary material available at https://doi. org/10.1186/s12870-021-02983-x.

Additional file 1: Figure S1. Pair-wise Pearson's correlation coefficients of the sequencing data of 60 samples. Figure S2. Dynamic expression patterns of genes during infection by real-time RT-PCR. Figure S3. Venn diagrams of specific and common DEGs responding to A. flavus in AF99 and AF32 (infection vs. mock-treatment) at different time points ( $\mathrm{a}, 0 \mathrm{hpi} ; \mathrm{b}, 0.5$ hpi; c,1.5 hpi; d, 3 hpi; e, 6 hpi). Figure S4. Venn diagram of up-regulated and down-regulated DEGs at different time points in AF99. Figure S5. Venn diagram of up-regulated and down-regulated DEGs at different time points in AF32. Figure S6. KEGG pathways enriched in down- and up-regulated genes responding to A. flavus in AF99 and AF32 (infection vs. mocktreatment) at different time points. Color depth represents the corrected $p$ value. Figure S7. MapMan-based visualization of an overview of the cellular response at the T4 stage in AF99(a) and AF32(b).

Additional file 2: Figure S8. KEGG analysis of the specific differentially expressed genes of fungal-inoculated kernels between AF99 and AF32. Color depth represents the degree of significance, as shown in the scale at the right (corrected $p$-value). Figure S9. Determination of softthresholding power ( $\beta$ ). a: scale-free topology fit index as a function of the soft-thresholding power, the red line indicates that $R^{2}$ is equal to 0.85. b: mean connectivity as a function of the soft-thresholding power. Figure S10. The sample dendrogram and trait heatmap. Lines: " 0 " for AF32 and "1" for AF99; Ino: "0" for mock and "1" for A. flavus inoculation; Time: "0, 0.5, 1.5, 3, 6" indicate the time after inoculation; RCO: "1" for the three biological repetitions of AF99 (mock-treated) at the T0 stage and "O" for other samples; R0: "1" for the three biological repetitions of AF99 (A. flavus inoculation)) at the T0 stage and "1" for other samples; SC0:"1" for the three biological repetitions of AF32 (mock-treated) at the T0 stage and " 0 " for all the other samples; S0:"1" for the three biological repetitions of AF32 (A. flavus inoculation) at the T0 stage and " 0 " for all the other samples; the rest are similar as "RCO", "RO", "SCO" and "SO". Figure S11. Scatterplots of gene significance versus module membership for the brown/RO(a), magenta/SC1(b), pink/S1(c), black/S2(d) and brown/S3(e), with correlations and $p$-values indicated. Figure S12. GO and KEGG pathway analysis of 110 hub genes identified in this study. a. GO analysis of 110 hub genes. Each circle represents an enrichment category, and the size of the circle indicates the number of genes. Detailed enrichment results are shown in Table S9 b. The top 20 associated KEGG pathways for the 110 hub genes. Figure S13. Heat map of 35 genes associated with the hypersensitive response and programmed cell death. Red indicates 
high expression, blue indicates low expression. Remove very low expression genes (average FPKM $<0.5$ ).

Additional file 3: Table S1. Detail information of sequencing reads from different sample groups.

Additional file 4: Table S2. List of primers used for Real-time RT-PCR.

Additional file 5: Table S3. Information of all genes in MapMan.

Additional file 6: Table S4. GO analysis of genes in the green module.

Additional file 7: Table S5. $G O$ analysis of genes in the yellow module.

Additional file 8: Table S6. GO analysis of genes in the brown module.

Additional file 9: Table S7. GO analysis of genes in the blue module.

Additional file 10: Table S8. List of hub genes identified in this study.

Additional file 11: Table S9. $\mathrm{GO}$ analysis of the 110 hub genes.

\section{Acknowledgements}

We thank Prof.DengDexiang (Yangzhou University, China) for extensive revisions to the manuscript.

\section{Authors' contributions}

Zhitong Yin and Huanhuan Liu contributed to the study design; Huanhuan Liu, Haofeng Wu, Yan Wang, Huan Wang and Saihua Chen contributed to the experimental execution and data analysis; Zhitong Yin, Huanhuan Liu and Haofeng Wu wrote the paper. All authors read and approved the final manuscript.

\section{Funding}

This work was supported by grants from the National Natural Science Foundation of China (31901551), National Science and Technology Major Project of the Ministry of Science and Technology of China (2018ZX0800904B), Jiangsu AgricultureScience and Technology Innovation Fund (CX (20) 1002), the Priority Academic Program Development of JiangsuHigher Education Institutions and Project of Special Funding for Crop Science Discipline Development (yzuxk202006).

\section{Availability of data and materials}

The raw data are available from the National Center for Biotechnology Information SRA Explorer (https://sra-explorer.info/?) under accession PRJNA691427.

\section{Declarations}

Ethics approval and consent to participate

Not applicable.

\section{Consent for publication}

Not applicable.

\section{Competing interests}

The authors declare that they have no competing interests.

Received: 7 January 2021 Accepted: 13 April 2021

Published online: 13 May 2021

\section{References}

1. Eaton DL, Gallagher EP. Mechanisms of aflatoxin carcinogenesis. Annu Rev Pharmacol. 1994;34(1):135-72.

2. Liu Y, Wu F. Global burden of aflatoxin-induced hepatocellular carcinoma: a risk assessment. Environ Health Perspect. 2010;118(6):818-24.

3. Campbell KW, White DG. Evaluation of corn genotypes for resistance to Aspergillus ear rot, kernel infection, and aflatoxin production. Plant Dis. 1995;79(10):1039.

4. Hamblin AM, White DG. Inheritance of resistance to aspergillus ear rot and aflatoxin production of corn from Tex6. Phytopathology. 2000;90(3):292.

5. Paul C, Naidoo G, Forbes A, Mikkilineni V, White D, Rocheford T. Quantitative trait loci for low aflatoxin production in two related maize populations. Theor Appl Genet. 2003;107(2):263-70.
6. Brooks TD, Williams WP, Windham GL, Willcox MC, Abbas HK. Quantitative trait loci contributing resistance to aflatoxin accumulation in the maize inbred Mp313E. Cropence. 2005;45(1):171-4.

7. Warburton ML, Brooks TD, Krakowsky MD, Shan XY, Windham GL, Williams WP. Identification and mapping of new sources of resistance to aflatoxin accumulation in maize. Crop Sci. 2009;49(4):1403-8.

8. Marilynl W, Thomasd B, Garyl W, Paul WW. Identification of novel QTL contributing resistance to aflatoxin accumulation in maize. Mol Breed. 2011; 27(4):491-9.

9. Mayfield KL, Murray SC, Rooney WL, Isakeit T, Odvody GA. Confirmation of QTL reducing aflatoxin in maize testcrosses. Crop Sci. 2011;51(6):2489.

10. Willcox MC, Davis GL, Warburton ML, Windham GL, Abbas HK, Betrán J, et al. Confirming quantitative trait loci for aflatoxin resistance from Mp313E in different genetic backgrounds. Mol Breed. 2013;32(1):15-26.

11. Yin Z, Wang Y, Wu F, Gu X, Bian Y, Wang Y, et al. Quantitative trait locus mapping of resistance to Aspergillus flavus infection using a recombinant inbred line population in maize. Mol Breed. 2014;33(1):39-49.

12. Mideros SX, Warburton ML, Jamann TM, Windham GL, Williams WP, Nelson RJ. Quantitative trait loci influencing mycotoxin contamination of maize: analysis by linkage mapping, characterization of near-isogenic lines, and meta-analysis. Crop Sci. 2014;54(1):127.

13. Dhakal R, Windham GL, Williams WP, Subudhi PK. Quantitative trait loci (QTL) for reducing aflatoxin accumulation in corn. Mol Breed. 2016; 36(12):164.

14. Warburton ML, Tang JD, Windham GL, Hawkins LK, Murray SC, Xu WW, et al. Genome-wide association mapping of Aspergillus flavus and aflatoxin accumulation resistance in maize. Crop Sci. 2015;55(5):1857-67.

15. Farfan IDB, De La Fuente GN, Murray SC, Isakeit T, Huang PC, Warburton M, et al. Genome Wide Association Study for Drought, Aflatoxin Resistance, and Important Agronomic Traits of Maize Hybrids in the Sub-Tropics. PLoS One. 2015;10:2.

16. Tang JD, Perkins A, Williams WP, Warburton ML. Using genome-wide associations to identify metabolic pathways involved in maize aflatoxin accumulation resistance. BMC Genomics. 2015;16:1.

17. Chen ZY, Brown RL, Damann KE, Cleveland TE. PR10 expression in maize and its effect on host resistance against Aspergillus flavus infection and aflatoxin production. Mol Plant Pathol. 2010;11(1):69-81.

18. Hawkins LK, Mylroie JE, Oliveira DA, Smith JS, Ozkan S, Windham GL, et al. Characterization of the maize chitinase genes and their effect on aspergillus flavus and aflatoxin accumulation resistance. PLoS One. 2015;10:6.

19. Majumdar R, Rajasekaran K, Sickler C, Lebar M, Musungu BM, Fakhoury AM, et al. The pathogenesis-related maize seed (PRms) gene plays a role in resistance to Aspergillus flavus infection and aflatoxin contamination. Front Plant Sci. 2017:8:1758.

20. Ogunola OF, Hawkins LK, Mylroie E, Kolomiets MV, Borrego E, Tang JD, et al. Characterization of the maize lipoxygenase gene family in relation to aflatoxin accumulation resistance. PLoS One. 2017;12:7.

21. Parish F, Williams WP, Windham GL, Shan XY. Differential Expression of Signaling Pathway Genes Associated With Aflatoxin Reduction Quantitative Trait Loci in Maize (Zea mays L.). Front Microbiol. 2019;10:2683.

22. Shi W, Yong-Soon P, Yang Y, Borrego EJ, Tom I, Xiquan G, et al. Seedderived ethylene facilitates colonization but not aflatoxin production by Aspergillus flavus in maize. Front Plant Ence. 2017;8(206):415.

23. Majumdar R, Minocha R, Lebar MD, Rajasekaran K, Long S, Carter-Wientjes C, et al. Contribution of maize polyamine and amino acid metabolism toward resistance against Aspergillus flavus infection and aflatoxin production. Front Plant Sci. 2019;10:692.

24. Jiang TB, Zhou BR, Luo M, Abbas HK, Kemerait R, Lee RD, et al. Expression analysis of stress-related genes in kernels of different maize (Zea mays L.) inbred lines with different resistance to aflatoxin contamination. Toxins. 2011;3(6):538-50.

25. Kelley RY, Williams WP, Mylroie JE, Boykin DL, Harper JW, Windham GL, et al. Identification of Maize Genes Associated with Host Plant Resistance or Susceptibility to Aspergillus flavus Infection and Aflatoxin Accumulation. PLoS One. 2012;7:5.

26. Dolezal AL, Shu XM, OBrian GR, Nielsen DM, Woloshuk CP, Boston RS, et al. Aspergillus flavus infection induces transcriptional and physical changes in developing maize kernels. Front Microbiol. 2014;5:384.

27. Shu XM, Livingston DP, Woloshuk CP, Payne GA. Comparative histological and transcriptional analysis of maize kernels infected with Aspergillus flavus and Fusarium verticillioides. Front Plant Sci. 2017;8:2075. 
28. Musungu BM, Bhatnagar D, Brown RL, Payne GA, OBrian G, Fakhoury AM, et al. A network approach of gene co-expression in the Zea mays/ Aspergillus flavus Pathosystem to map host/pathogen interaction pathways. Front Genet. 2016;7:206.

29. Musungu B, Bhatnagar D, Quiniou S, Brown RL, Geisler M. Use of dual RNAseq for systems biology analysis of Zea mays and Aspergillus flavus interaction. Front Microbiol. 2020;11:1.

30. Chao W, Qin Y, Weixiang Y, Li Y, Guo D. A transposon-directed epigenetic change in ZmCCT underlies quantitative resistance to Gibberella stalk rot in maize. New Phytol. 2017;215(4):1503.

31. Yao L, Li Y, Ma C, Du LTF, Xu M. Combined genomeide association study and transcriptome analysis reveal candidate genes for resistance to Fusarium ear rot in maize. J Integr Plant Biol. 2020;62(10):1535.

32. Orlowska E, Fiil A, Kirk HG, Llorente B, Cvitanich C. Differential gene induction in resistant and susceptible potato cultivars at early stages of infection by Phytophthora infestans. Plant Cell Rep. 2012;31(1):187-203.

33. Zhu JH, Kapoor A, Sridhar W, Agius F, Zhu JK. The DNA glycosylase/lyase ROS1 functions in pruning DNA methylation patterns in Arabidopsis. Curr Biol. 2007;17(1):54-9.

34. Le TN, Schumann U, Smith NA, Tiwari S, Au PCK, Zhu QH, et al. DNA demethylases target promoter transposable elements to positively regulate stress responsive genes in Arabidopsis. Genome Biol. 2014;15:9.

35. Sanchez AL, Stassen JHM, Furci L, Smith LM, Ton J. The role of DNA (de)methylation in immune responsiveness of Arabidopsis. Plant J. 2016; 88(3):361-74.

36. Gong ZH, Morales-Ruiz T, Ariza RR, Roldan-Arjona T, David L, Zhu JK. ROS1, a repressor of transcriptional gene silencing in Arabidopsis, encodes a DNA glycosylase/lyase. Cell. 2002;111(6):803-14.

37. Bies-Etheve N, Pontier D, Lahmy S, Picart C, Vega D, Cooke R, et al. RNAdirected DNA methylation requires an AGO4-interacting member of the SPT5 elongation factor family. EMBO Rep. 2009;10(6):649-54.

38. He XJ, Hsu YF, Zhu SH, Wierzbicki AT, Pontes O, Pikaard CS, et al. An effector of RNA-directed DNA methylation in Arabidopsis is an ARGONAUTE 4-and RNA-binding protein. Cell. 2009;137(3):498-508.

39. Li XD, Xia B, Jiang YM, Wu QS, Wang CY, He LS, et al. A new pathogenesisrelated protein, LrPR4, from Lycoris radiata, and its antifungal activity against Magnaporthe grisea. Mol Biol Rep. 2010;37(2):995-1001.

40. Svensson B, Svendsen I, Hoejrup P, Roepstorff P, Ludvigsen S, Poulsen FM. Primary structure of barwin: a barley seed protein closely related to the Cterminal domain of proteins encoded by wound-induced plant genes. Biochemistry. 1992;31(37):8767-70.

41. Dong ZB, Jiang $C$, Chen XY, Zhang T, Ding L, Song WB, et al. Maize LAZY1 mediates shoot Gravitropism and inflorescence development through regulating auxin transport, auxin signaling, and light response. Plant Physiol. 2013;163(3):1306-22.

42. Xie YR, Chen ZY, Brown RL, Bhatnagar D. Expression and functional characterization of two pathogenesis-related protein 10 genes from Zea mays. J Plant Physiol. 2010;167(2):121-30.

43. Corpas FJ, Barroso JB. Lead-induced stress, which triggers the production of nitric oxide (NO) and superoxide anion (O-2(center dot-)) in Arabidopsis' peroxisomes, affects catalase activity. Nitric Oxide-Biol Ch. 2017;68:103-10.

44. Piacentini D, Corpas FJ, D'Angeli S, Altamura MM, Falasca G. Cadmium and arsenic-induced-stress differentially modulates Arabidopsis root architecture, peroxisome distribution, enzymatic activities and their nitric oxide content. Plant Physiol Biochem. 2020;148:312-23.

45. Hawkins LK, Warburton ML, Tang JD, Tomashek J, Oliveira DA, Ogunola OF, et al. Survey of Candidate Genes for Maize Resistance to Infection by Aspergillus flavus and/or Aflatoxin Contamination. Toxins. 2018;10:2.

46. Luo M, Brown RL, Chen ZY, Menkir A, Yu JJ, Bhatnagar D. Transcriptional profiles uncover Aspergillus flavus-induced resistance in maize kernels. Toxins. 2011;3(7):766-86.

47. Greenberg JT, Yao N. The role and regulation of programmed cell death in plant-pathogen interactions. Cell Microbiol. 2004;6(3):201-11.

48. Wang $\mathrm{H}$, Lin J, Chang $\mathrm{YH}$, Jiang CZ. Comparative transcriptomic analysis reveals that ethylene/H2O2-mediated hypersensitive response and programmed cell death determine the compatible interaction of sand pear and Alternaria alternata. Front Plant Sci. 2017;8:195.

49. Cao Z, Kapoor K, Li L, Banniza S. Interactive gene expression patterns of susceptible and resistant Lens ervoides recombinant inbred lines and the Necrotroph Ascochyta lentis. Front Microbiol. 2020;11:1259.
50. Zhu XL, Lu CG, Du LP, Ye XG, Liu X, Coules A, et al. The wheat NB-LRR gene TaRCR1 is required for host defence response to the necrotrophic fungal pathogen Rhizoctonia cerealis. Plant Biotechnol J. 2017;15(6):674-87.

51. Kouzai $Y$, Kimura M, Watanabe M, Kusunoki K, Osaka D, Suzuki T, et al. Salicylic acid-dependent immunity contributes to resistance against Rhizoctonia solani, a necrotrophic fungal agent of sheath blight, in rice and Brachypodium distachyon. New Phytol. 2018;217(2):771-83.

52. Frederikke G. Malinovsky, Peter Brodersen, Berthe Katrine Fiil, Lea Vig McKinney, Stephan Thorgrimsen, Martina Beck, H. Bjørn Nielsen, Stefano Pietra, Cyril Zipfel, Silke Robatzek, Morten Petersen, Daniel Hofius, John Mundy, Mohammed Bendahmane, (2010) Lazarus1, a DUF300 Protein, Contributes to Programmed Cell Death Associated with Arabidopsis acd11 and the Hypersensitive Response. PLoS ONE 5 (9):e12586.

53. Malinovsky FG, Brodersen P, Fiil BK, McKinney LV, Thorgrimsen S, Beck M, Nielsen HB, Pietra S, Zipfel C, Robatzek S, Petersen M, Hofius D, Mundy J, Bendahmane M. Lazarus1, a DUF300 Protein, Contributes to Programmed Cell Death Associated with Arabidopsis acd 11 and the Hypersensitive Response. PLoS ONE. 2020;5(9):e12586.

54. Morrissey JP, Osbourn AE. Fungal resistance to plant antibiotics as a mechanism of pathogenesis. Microbiol Mol Biol R. 1999;63(3):708.

55. Christensen SA, Sims J, Vaughan MM, Hunter C, Block A, Willett D, et al. Commercial hybrids and mutant genotypes reveal complex protective roles for inducible terpenoid defenses in maize. J Exp Bot. 2018;69(7): 1693-705.

56. Huffaker A, Kaplan F, Vaughan MM, Dafoe NJ, Ni XZ, Rocca JR, et al. Novel acidic Sesquiterpenoids constitute a dominant class of pathogen-induced Phytoalexins in maize. Plant Physiol. 2011;156(4):2082-97.

57. Balmer D, de Papajewski DV, Planchamp C, Glauser G, Mauch-Mani B. Induced resistance in maize is based on organ-specific defence responses. Plant J. 2013;74(2):213-25.

58. Schmelz EA, Huffaker A, Sims JW, Christensen SA, Lu X, Okada K, et al. Biosynthesis, elicitation and roles of monocot terpenoid phytoalexins. Plant J. 2014;79(4):659-78.

59. Christensen SA, Huffaker A, Kaplan F, Sims J, Ziemann S, Doehlemann G, et al. Maize death acids, 9-lipoxygenase-derived cyclopente(a)nones, display activity as cytotoxic phytoalexins and transcriptional mediators. P Natl Acad Sci USA. 2015;112(36):11407-12.

60. Ding YZ, Huffaker A, Kollner TG, Weckwerth P, Robert CAM, Spencer JL, et al. Selinene volatiles are essential precursors for maize defense promoting fungal pathogen resistance. Plant Physiol. 2017;175(3):1455-68.

61. Ding Y, Weckwerth PR, Poretsky E, Murphy KM, Sims J, Saldivar E, et al. Genetic elucidation of interconnected antibiotic pathways mediating maize innate immunity. Nat Plants. 2020;6(11):1375.

62. Deng DX, Jiang SX, Wang YJ, Bian YL, Chen JJ, Bo J. Genetic analysis of the resistance to Aspergillus flavus infection in maize (Zea mays L.). J Integr Agric (in chinese). 2009;8(6):761-5.

63. Dexiang D. Identification of maize germplasm resistant to Aspergillus flavus. J Plant Gen Resourc. 2002;3:22.

64. Zhang Y, Cui M, Zhang J, Zhang L, Li C, Kan X, et al. Confirmation and fine mapping of a major QTL for aflatoxin resistance in maize using a combination of linkage and association mapping. Toxins. 2016:8(9):258.

65. Li RQ, Li YR, Kristiansen K, Wang J. SOAP: short oligonucleotide alignment program. Bioinformatics. 2008;24(5):713-4.

66. Kim D, Langmead B, Salzberg SL. HISAT: a fast spliced aligner with low memory requirements. Nat Methods. 2015;12(4):357-60.

67. Li B, Dewey CN. RSEM: accurate transcript quantification from RNA-Seq data with or without a reference genome. Bmc Bioinformatics. 2011;12:1.

68. Love MI, Huber W, Anders S. Moderated estimation of fold change and dispersion for RNA-seq data with DESeq2. Genome Biol. 2014;15:12.

69. Bindea G, Mlecnik B, Hackl H, Charoentong P, Tosolini M, Kirilovsky A, et al. ClueGO: a Cytoscape plug-in to decipher functionally grouped gene ontology and pathway annotation networks. Bioinformatics. 2009;25(8):1091-3.

70. Thimm O, Blasing O, Gibon Y, Nagel A, Meyer S, Kruger P, et al. MAPMAN: a user-driven tool to display genomics data sets onto diagrams of metabolic pathways and other biological processes. Plant J. 2004;37(6):914-39.

71. Zhang B, Horvath S. A general framework for weighted gene co-expression network analysis. Stat Appl Genet Mol. 2005:4:1.

72. Langfelder P, Horvath S. WGCNA: an R package for weighted correlation network analysis. Bmc Bioinform. 2008;9:1.

73. Garg R, Singh VK, Rajkumar MS, Kumar V, Jain M. Global transcriptome and coexpression network analyses reveal cultivar-specific molecular signatures 
associated with seed development and seed size/weight determination in chickpea. Plant J. 2017;91(6):1088-107.

74. Shannon P, Markiel A, Ozier O, Baliga NS, Wang JT, Ramage D, et al.

Cytoscape: a software environment for integrated models of biomolecular interaction networks. Genome Res. 2003;13(11):2498-504.

75. Chen CJ, Chen H, Zhang Y, Thomas HR, Frank MH, He YH, et al. TBtools: an integrative toolkit developed for interactive analyses of big biological data. Mol Plant. 2020;13(8):1194-202

\section{Publisher's Note}

Springer Nature remains neutral with regard to jurisdictional claims in published maps and institutional affiliations.

Ready to submit your research? Choose BMC and benefit from:

- fast, convenient online submission

- thorough peer review by experienced researchers in your field

- rapid publication on acceptance

- support for research data, including large and complex data types

- gold Open Access which fosters wider collaboration and increased citations

- maximum visibility for your research: over $100 \mathrm{M}$ website views per year

At $\mathrm{BMC}$, research is always in progress.

Learn more biomedcentral.com/submissions 